\title{
A Brief History of Excitable Map-Based Neurons and Neural Networks
}

\author{
M. Girardi-Schappo ${ }^{\mathrm{a}}$, M. H. R. Tragtenberg ${ }^{\mathrm{a}}$, O. Kinouchi ${ }^{\mathrm{b}}$ \\ ${ }^{a}$ Departamento de Física, Universidade Federal de Santa Catarina, 88040-900, \\ Florianópolis, Santa Catarina, Brazil \\ ${ }^{b}$ Departamento de Física, FFCLRP, Universidade de São Paulo, 14040-900, Ribeirão \\ Preto, São Paulo, Brazil
}

\begin{abstract}
This review gives a short historical account of the excitable maps approach for modeling neurons and neuronal networks. Some early models, due to Pasemann (1993), Chialvo (1995) and Kinouchi and Tragtenberg (1996), are compared with more recent proposals by Rulkov (2002) and Izhikevich (2003). We also review map-based schemes for electrical and chemical synapses and some recent findings as critical avalanches in map-based neural networks. We conclude with suggestions for further work in this area like more efficient maps, compartmental modeling and close dynamical comparison with conductance-based models.
\end{abstract}

Keywords: Difference equations, Neuron Models, Coupled Map Lattices, Neural Networks, Excitable Dynamics, Excitable Media, Bursting, Map-based Neuron, Map-based Synapses

\section{Introduction}

The number of neurons in the human brain (86 billions Azevedo et al. 2009)) is near six times the number of trees in Amazonia. So, brain mod- 
elers must not forget that their job is comparable to modeling patches of Amazonia, a staggering task. Since well developed models for single neurons already exist (Bower and Beeman, 2003, Carnevale and Hines, 2006), with complex dendritic geometry and tens of equations and parameters (Dayan and Abbott, 2001), it is not obvious what modeling level we should use in general.

As the proverbial forest not seen because of the trees, the detailed study of singular neurons is an interesting subject per se but perhaps not necessary to understand the macroscopic dynamics and function of neuronal networks. Indeed, neuronal networks present collective phenomena, like synchronization, waves and avalanches, with regimes separated by global bifurcations or phase transitions, that cannot be studied in small neuronal populations. The history of neuronal networks modeling is marked by this trade-off between analytical/computational tractability and biological realism.

Since the connection between neurons is only sensitive to the action potentials that arrive at (electrical or chemical) synapses, the important thing is to model the dynamics of these action potentials (their frequency or interspike intervals, if they come in bursts or single events etc.). The emphasis in modeling the transmembrane voltage dynamical behavior is called a phenomenological approach (in contrast to a mechanistic or biophysical approach), leads to a class of neuron models where map-based neurons are a new and promising tool. This paper gives a brief account of the pionnering proposals of neuronal maps due to Chialvo (1995), Pasemann (1993, 1997), Kinouchi and Tragtenberg (1996) and Kuva et al. (2001) and compare them with more recent proposals due to Rulkov 2001, 2002) and Izhikevich (2003); 
Izhikevich and Hoppensteadt (2004).

There is two main routes to achieve map-based neuron models with realistic dynamical properties. The first one is to start from Hodgkin-Huxley (HH) type models, composed by coupled nonlinear ordinary differential equations (ODE) which are already a simplification (due to spatial discretization) of full partial differential equations that describes the neuron membrane. Computational neuroscience models based on the $\mathrm{HH}$ formalism, called conductancebased neurons, is a well developed subject (Dayan and Abbott, 2001), but suffers from some limitations (de Schutter, 2010):

- The HH-type models consist in several nonlinear coupled EDOs: the simulation of a single neuron is orders of magnitude more costly than simplified neuron models;

- The biophysical data for constraining the parameter values (like capacitances, axial resistances, density of ion channels, etc.) is scarce and often obtained from diverse preparations (different animals, in vitro experiments etc.). Most of the parameter ranges used in simulations are simply informed guesses.

- The remaining parameter space of these models is huge and suffer from the so called curse of dimensionality (Bellman, 2003). It is very costly to trace full phase diagrams, since with $P$ parameters, for example, we can have $P(P-1)$ parameter planes. The minimal model of HodgkinHuxley, with only two active ion channels, has at least $P=40$ parameters (Dayan and Abbott, 2001).

- The set of parameters to be used for reproducing a given firing pattern 
is subdetermined. This means that the same dynamical behavior can be achieved by different sets of parameters. Adjusting these parameters to the known neuron behavior is susceptible of overfitting: the model reproduces the given data but do not generalizes well, for example, for different input situations.

In order to deal with these drawbacks, we may opt to reproduce the dynamical behavior of neurons instead of reproducing the involved biophysical mechanisms (mechanistic modeling). Starting from a complicated HH-model, perhaps even a multicompartimental model, we can perform a sequence of simplifications more or less justified in order to obtain simpler models with fewer equations and lumped parameters (de Schutter, 2010). Examples of these reduced ODE's based models are the FitzHugh-Nagumo excitable neuron (FitzHugh, 1955; Nagumo et al., 1962), the Hindmarsh-Rose bursting neuron (Hindmarsh and Rose, 1984) and the Izhikevitch model (Izhikevich, 2003). If we numerically integrate these ODEs with the Euler method with a large time step, we can arrive to maps with similar dynamical properties as the original systems (Rulkov, 2002, Izhikevich, 2004).

Phenomenological modeling can start the other way around. This occurs because the phenomena to be studied sets the level of modeling. Continuing with our forest modeling analogy, if our interest is to study a single tree (or neuron), a biophysical HH-like modeling is desirable. But if we want to understand, say, the propagation of a forest fire, the modeling of the tree biophysics is mostly immaterial, and trees could be represented by sites with two states $(0=$ normal, $1=$ burnt) (Christensen et al., 1993). In the same vein, McCulloch and Pitts (1943) proposed a binary threshold neuron, 
whose state is given by $0=$ rest and $1=$ firing. With this method, one starts with discrete time systems and searches for increasing complexity until achieve dynamical models that reproduce the full phenomenology of neuronal dynamics.

Both approachs tend to converge at a middle ground formalism: dynamical systems with discrete space and discrete time, but with continuous state variables, that is, dynamical maps (Ibarz et al., 2011). Neuronal networks composed by these maps will be an instance of coupled maps lattices (CMLs) (Kaneko, 1993. 1994). In this paper, we review the early proposals of mapbased neuron models and the coupling schemes used to create such coupled maps networks. We also suggest some unexplored research topics that could be examined both with conductance-based neurons and map-based neurons, in order to stimulate computational neuroscientists to use both approachs in a synergetic way.

This review is intended to organize the map-based neuron models in sequential time (Sec. 2), highlighting two families of map-based modeling: (I) back from McCulloch and Pitts (1943) approach to the Kinouchi and Tragtenberg (1996) and its extension (Kuva et al., 2001) and (II) back from Chialvo (1995) to Rulkov (2002) and Izhikevich (2003). Then, we perform a short computational comparison of the main neuronal models (Sec. 3). The main purpose of Sec. 4 is to neatly list the most relevant couplings which may be used to link maps into networks, only pointing to the most prominent results obtained with them. We finally terminate the review with some important remarks in Sec. 5. 


\section{History of Map-based Neurons}

This section is devoted to draw a line which connects the early modeling of neurons, as state machines, to the most recent and powerful models, which are dynamical systems on their own, presenting their most relevant features and reviewing some models that are still not well known, although they have been used recently to model neural networks.

The generalized mathematical form of any map is:

$$
\vec{x}(t+1)=\vec{F}[\vec{x}(t)],
$$

where $\vec{F}: \mathbb{R}^{n} \rightarrow \mathbb{R}^{n}$ is any vector function and we are assuming that the

curve given by the set of values $\{\vec{x}(t)\}$ defines the temporal evolution of some quantity. In the case of neurons, each component of the vector $\vec{x}(t)$ accounts for a relevant neuronal quantity.

Generally, the first component, $x^{1}(t)$, is the membrane potential (the fast variable) and the second component, $x^{2}(t)$, may be the slow inward and outward currents or an auxiliary variable. When present, the third component, $x^{3}(t)$, accounts for the slow currents. For the sake of simplicity, we define $x(t) \equiv x^{1}(t), y(t) \equiv x^{2}(t)$ and $z(t) \equiv x^{3}(t)$ throughout this paper. The subscript index is intended to identify elements of a network.

\subsection{Early History}

McCulloch and Pitts (1943) formal neuron can be viewed, if coupled to $N$ presynaptic neurons with parallel update, as a discrete time dynamical system (Little, 1974):

$$
x_{i}(t+1)=H\left(\sum_{j \neq i}^{N} J_{i j} x_{j}(t)-\theta+I_{i}(t)\right)
$$


where the Heaviside (step) function gives $H(y)=1$ if $y \geq 0$ (zero otherwise), $I_{i}(t)$ is the external input and $\theta$ is a firing threshold.

In the statistical physics community, due to symmetry motivations, it is common to scale the neuron output as a sign function with values \pm 1 , that is, $S(y)=2 H(y)-1$.

The isolated McCulloch-Pitts neuron has no intrinsic dynamics (notice that the input sum is over $j \neq i$ ). However we can introduce a dependence on the past values of its variable, as in the Caianello (1961) equations:

$$
x_{i}(t+1)=H\left[\sum_{n=0}^{\tau}\left(W_{i}^{(n)} x_{i}(t-n)+\sum_{j \neq i}^{N} J_{i j}^{(n)} x_{j}(t)\right)-\theta+I_{i}(t)\right],
$$

where now the $W_{i}^{(n)}$ parameters weight the contributions of the delayed $x_{i}(t-$ $n$ ) values within a memory window $\tau$. So, the isolated neuron $\left(J_{i j}=0\right)$ can present interesting dynamical behavior.

Indeed, Nagumo and Sato (1972) studied an isolated formal neuron with an exponential decay of refractory influence, that is,

$$
x(t+1)=H\left(\sum_{n=0}^{\tau} W^{(n)} x(t-n)-\theta+I(t)\right),
$$

where $W^{(n)}=-\alpha w^{n}$, with a decay constant $w$. It has been shown that almost all solutions of the Nagumo-Sato model are periodic and form a complete devil staircase - chaotic solutions lie at the complementary zero measure cantor set (Aihara and Suzuki, 2010).

Since the $x(t)$ variable has a discrete set of values, this kind of formal neurons corresponds to cellular automata, not to continuous maps. But starting from the decade of 1980, modelers substituted the discontinuous 
step or sign functions by continuous ones:

$$
x(t+1)=F\left(\sum_{n=0}^{\tau} W^{(n)} x(t-n)-\theta+I(t)\right),
$$

where, for example, the transfer function is a logistic function $F(y)=[1+$ $\exp (-\gamma y)]^{-1}$ or a hyperbolic tangent $F(y)=\tanh (y / T)$ Hopfield, 1984 , Aihara et al., 1990), where $\gamma$ and $1 / T$ are gain parameters. Now, the $x(t+1)=F(x(t), x(t-1), \ldots, x(t-\tau))$ is a $\tau+1$ dimensional map. Fig. 1 illustrates the differences between Eqs. 2 and 5 for $\tau=1$.

The case $\tau=0$ with a logistic function $F(y)$, that is, $x(t+1)=F(\gamma(x(t)+I(t)-\theta))$ was examined by Pasemann (1993). In the plane $(\gamma, H=I-\theta)$ it presents only three behaviors: a fixed point phase, a period two phase and a bistability (two fixed points) phase (Pasemann, 1993; Kinouchi and Tragtenberg, 1996). A chaotic version of this neuron map has also been studied by Pasemann (1997).

Due to a lucky accident, the case $\tau=1$ implements a powerful excitable element with a very rich behavior. We call this system a second order dy-

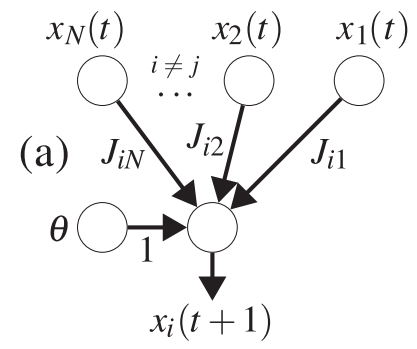

(b)

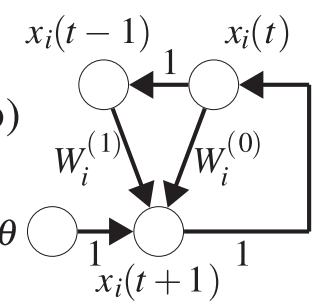

Figure 1: (a) Scheme of a single-layer perceptron (Eq. 2) and (b) the so-called dynamical perceptron (Eq. 5 with $\tau=1$ ). In both cases, the output $x_{i}(t+1)$ may be calculated by a continuous function or by a discret step function. 
namical perceptron or the Kinouchi and Tragtenberg 1996) map (also known in the statistical mechanics literature as the YOS map (Yokoi et al., 1985, Tragtenberg and Yokoi, 1995)). This excitable neuron model is reviewed in the next section.

So far all the models follow the same principle: they are built directly from discrete time dynamics. However, neuronal models can be built the other way around: starting from continuous time differential equations, like the HH model, and then simplify them to keep only their wanted features. A result of this kind of simplification is proposed by Chialvo (1995) to study excitable systems (and, in particular, neural excitability):

$$
\begin{aligned}
& x(t+1)=[x(t)]^{2} \exp [y(t)-x(t)]+I(t) \\
& y(t+1)=a y(t)-b x(t)+c
\end{aligned}
$$

where $a, b$ and $c$ are parameters and $I(t)$ may account for a bias membrane potential or external input. Chialvo inspired more recent works, like the Rulkov (2002) and the Izhikevich (2003) models, both studied in Sec. 2.3

\subsection{KT and $K T z$ Maps}

The case $\tau=1$ with $\mathrm{F}(y)=\tanh (y)$ was extensively studied in the context of Statistical Mechanics and resulted in many phase diagrams (Tragtenberg and Yokoi, 1995). In order to build on these results, Kinouchi and Tragtenberg (1996) imposed, in Eq. 5, the change of parameters $K \equiv-W^{(1)} / W^{(0)}$, $T \equiv 1 / W^{(0)}$ and $H \equiv\left(\theta+W^{(0)}+W^{(1)}\right) / W^{(0)}$. Rewriting Eq. 5 leads us to the KT model:

$$
\begin{aligned}
& x(t+1)=\tanh \left(\frac{x(t)-K y(t)+H+I(t)}{T}\right), \\
& y(t+1)=x(t),
\end{aligned}
$$


where $-1<x(t)<+1$ represents the actual membrane potential of the neuron at time $t$-measured in timesteps (ts). The term $I(t)$ corresponds to an external input. In section 4 the coupling is done via $I(t)$ as well. Both $x(t), I(t)$ and $t$ may be conveniently rescaled to any unit system, like mili-Volts or mili-seconds (ms). The authors assumed $1 \mathrm{ts}=0.1 \mathrm{~ms}$ whilst the membrane potential may be rescaled to fit a Hodgkin-Huxley model, for instance, by relating the value of the membrane potential at the fixed point and at the peak of the spike in both models.

The authors reinterpreted the statistical mechanical phase diagrams, as shown in Fig. 2, proposing that the relevant neuronal behaviors are in the parameter subspace given by $0.5 \leq K \leq 1$, with $T$ and $H$ near the phase transitions from fixed point to oscillatory behavior (i.e. fast spiking).

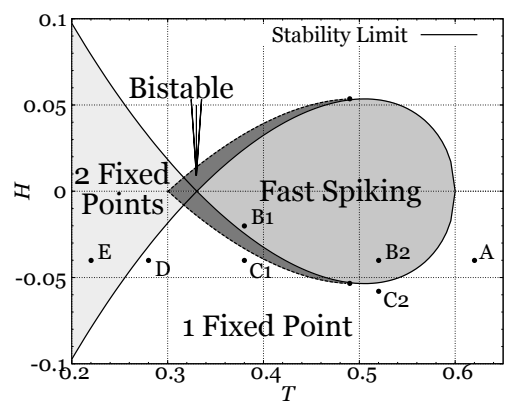

Figure 2: KT model phase diagram for $K=0.6$. It allowed authors to list five different neuronal behaviors, labeled from A to E, discussed in the text. The Bistable region corresponds to coexistence of oscillatory with fixed point behaviors.

The stability limit of the model, the curve $H_{s}(K, T)$, separating the oscillating and the fixed point phases may be calculated via a simple linear 
stability analysis, which yields:

$$
H_{s}^{ \pm}(K, T)=T \operatorname{atanh}\left(x_{s}^{*}\right)-(1-K) x_{s}^{*},
$$

where $x_{s}^{*}$ is the fixed point over the line $H_{s}(K, T)$, given by:

$$
x_{s}^{*}=\left\{\begin{array}{ll} 
\pm \sqrt{1-\frac{T}{1-K}} & , \text { if } 0<K \leq 0.5 \\
\pm \sqrt{1-\frac{T}{K}} & , \text { if } 0.5<K \leq 1
\end{array} .\right.
$$

Differently from the statistical mechanical model, the KT model may be extended for $T<0$ and $K<0$. It would result in a slightly different $x_{s}^{*}$, although the analysis would keep the same (Kinouchi and Tragtenberg, 1996). Thus, we will focus on positive parameters.

Notice that the parameter $H$ may be redefined as $\tilde{H}=H+I(t)$, so the effect of an external input is to drag the solution $x(t)$ for Eq. 7 along any vertical line (with fixed $T$ ) on Fig. 2. This is the basic mechanism of the KT model excitability, in which $I(t)$ is such that $H+I(t)>H_{s}(K, T)$ - assuming the neuron is adjusted below de bubble of Fig. 2. This allows the authors to list five distinct neuronal behaviors (labeled from A to E in Fig. 2). These behaviors are present in Hodgkin-Huxley-like neurons and in experimental setups (Kinouchi and Tragtenberg, 1996):

- Neuron A: Silent neuron - it will not fire any action potential, regardless the external stimulus intensity - no bifurcation occurs;

- Neuron B (1 and 2): Pacemaker neuron - it is an autonomous oscillator, although external stimulation may bring the neuron to outside of the bulb in Fig. 2 temporarily via a Subcritical Neimarck-Sacker bifurcation (B1) or a Supercritical Neimarck-Sacker bifurcation (B2); 
- Neuron C (1 and 2): Excitable neuron - it will fire only for a stimulus greater than some threshold; the stimulus takes the neuron inside the bulb in Fig. 2 through a Subcritical Neimarck-Sacker Bifurcation (C1) or a Supercritical Neimarck-Sacker Bifurcation (C2). If a neuron C1 is adjusted over the Bistable region, then external stimuli may switch between fast spiking and fixed point response (phenomenon known as pacemaker activity annihilation);

- Neuron D: presents a single fixed point; however, a strong enough external current, $I(t)$, may transform it into a bistable neuron with two stable fixed points;

- Neuron E: presents two stable fixed points even without external input; the neuron may switch between states through some stimulation $I(t)$.

Type C1 neuron, specially, presents a rich repertoire of excitable responses (see Fig. 33). It also gave the authors insights about the mathematical nature of transient oscillations - not explained, but experimentally observed by Morris and Lecar (1981): all behaviors of type C1 are achieved because the neuron lies near a bifurcation point.

KT model also presents a well defined excitability, as shown in the last panel of Fig. 3 - in which the neuron receives a delta current input $I(t)=$ $I_{0} \delta_{t, t_{0}}$ in $t_{0}=0$ with increasing intensity $I_{0}$. The circle-dash line shows the approximate threshold $I_{s}$ : any stronger stimulus causes a spike. The nullclines of the model are of the same shape as those of the FitzHughNagumo model (see Fig. 4).

Besides excitability, KT model also presents regular and chaotic au- 

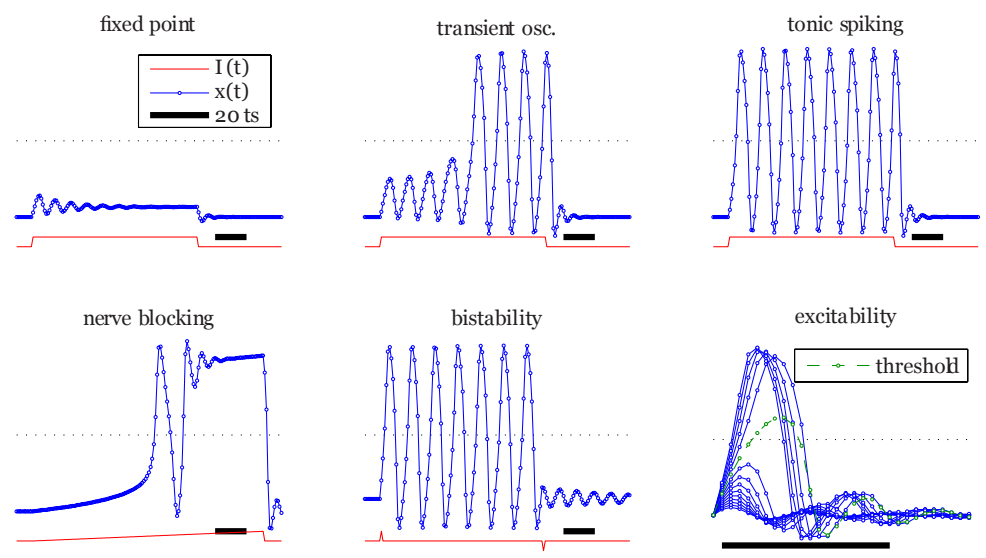

Figure 3: Excitable response of KT type $\mathrm{C} 1$ model. The solution $x(t)(-\circ-)$ corresponds only to the circle points (the lines are just guides so one can follow the sequence in which the map evolves through time). Solid line is external input and the bolder line segment corresponds to 20 ts.

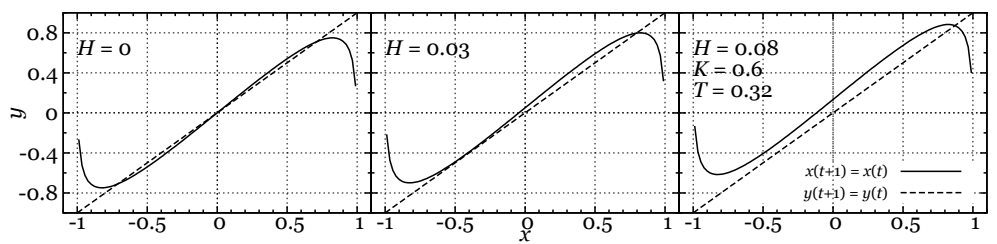

Figure 4: Effect of varying parameter $H$ on the nullclines of the KT model. Notice the similarity with the nullclines of the FitzHugh-Nagumo model.

tonomous behavior (Fig. 50. The authors also proposed a mechanism to generate bursts based on the Hindmarsh-Rose model (Kinouchi and Tragtenberg, 1996): by letting the parameter $H \equiv z(t)$, in Eq. 7, oscillate slowly in time, with

$$
z(t+1)=(1-\delta) z(t)-\lambda(x(t)-y(t))^{2},
$$

where $\delta$ and $\lambda$ are paremeters that control the inward and the outward ionic 
currents, respectively. This modification allows the neuron to go back and forth inside the bulb in Fig. 2. This mechanism generates bursts when the transition induced by the $z(t)$ dynamics is via a Subcritical Hopf Bifurcation (i.e. through the bistable region in Fig. 2).
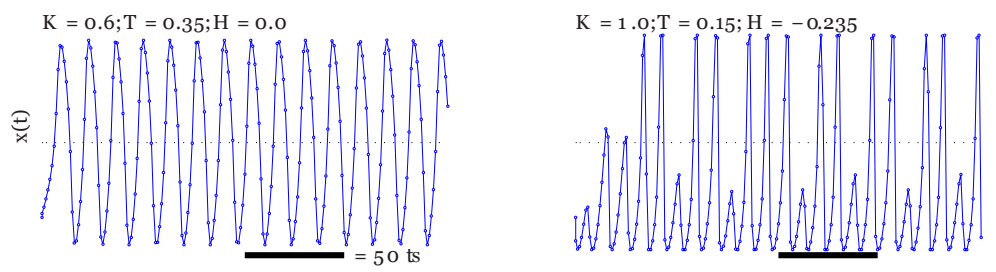

Figure 5: Left: Fast spiking behavior (inside the bulb in Fig. 22. Right: Chaotic spiking.

Furthermore, Veiga and Tragtenberg (2001) showed that KT model, Eq. 7. may present stochastic resonance (which is the occurrence of spiking due to subthreshold stochastic stimulation). Beyond the usual stochastic resonance, the authors achieved autonomous and aperiodic stochastic resonance as well.

Kuva et al. (2001) modified the $z(t)$ dynamics (from Eq. 10) of the three variables KT model into a simpler, yet burster, version of the neuron, which we now name the KTz model. The equations are:

$$
\begin{aligned}
& x(t+1)=\tanh \left(\frac{x(t)-K y(t)+H+I(t)}{T}\right), \\
& y(t+1)=x(t), \\
& z(t+1)=(1-\delta) z(t)-\lambda\left(x(t)-x_{R}\right),
\end{aligned}
$$

where $x(t)$ is the membrane potential of the neuron, $y(t)$ is a recovery variable and $z(t)$ is the slow total ionic current. The $K$ and $T$ parameters control the fast spiking dynamics, as explained above. The parameter $\delta$ is the inverse recovery time of $z(t), \lambda$ and $x_{R}$ control the slow spiking and bursting dynam- 
ics Kuva et al. (2001). In practice, though, $\delta, \lambda$ and $x_{R}$ roles are illustrated in Fig. 6.

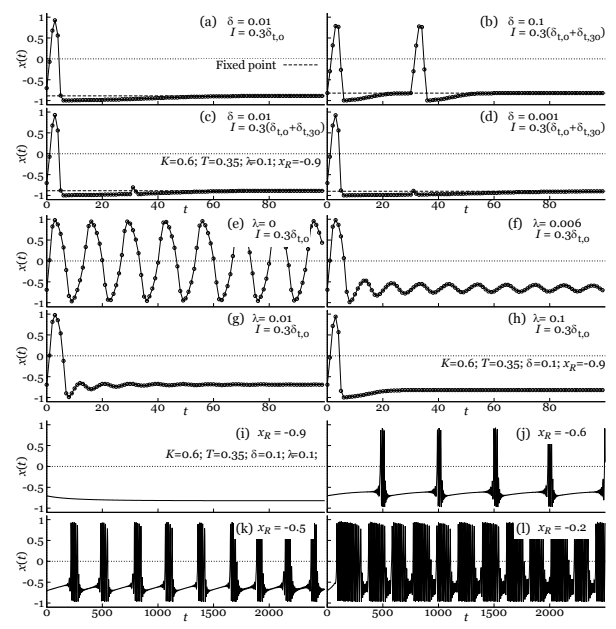

Figure 6: (a) to (d): $\delta$ control the length of the refractory period; (e) to (h): $\lambda$ control the damping of oscillations; (i) to (l): $x_{R}$ control the bursting dynamics. Parameters' values are listed in the panels.

KTz autonomous behaviors are listed in Fig. 8 whilst the excitable behaviors are in Fig. 9. A phase diagram for $\lambda=\delta=0.001$ and $K=0.6$ is given by Kuva et al. (2001) (see Fig. 7). The fixed point stability of this model has been studied by Copelli et al. (2004).

We classified the KTz excitable behaviors according to Izhikevich and Hoppensteadt (2004), searching for qualitative similarities between their model and the KTz model. The latter presented 15 out of the 20 excitable behaviors described by Izhikevich and Hoppensteadt (2004).

Although it does not present every excitable behavior, it is a minimal model, based on only five parameters $\left(K, T, \delta, \lambda\right.$ and $\left.x_{R}\right)$. Besides, the $\mathrm{KTz}$ 


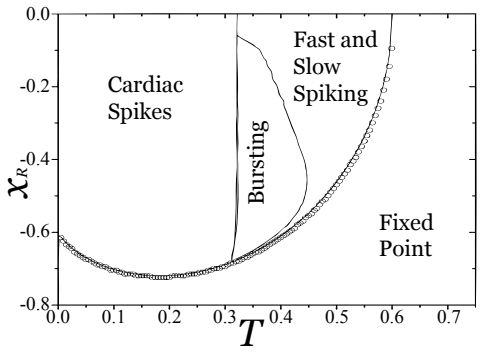

Figure 7: Detailed phase diagram for the $\mathrm{KTz}$ model $(\lambda=\delta=0.001$ and $K=0.6)$.

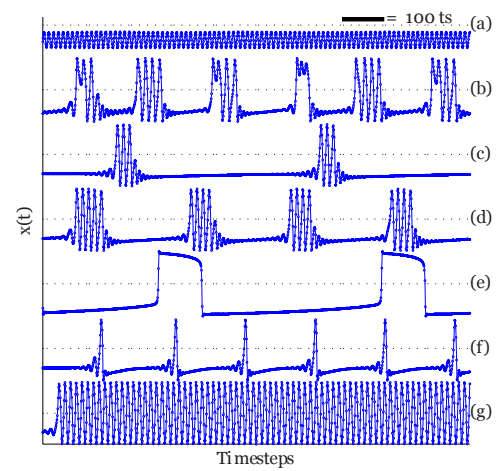

Figure 8: Examples of KTz model autonomous behaviors. (a) Subthreshold oscillations; (b) Chaotic bursting; (c) Slow bursting; (d) Fast bursting; (e) Cardiac spikes; (f) Slow spiking; (g) Fast spiking.

map (Eq. 11) has no singularities and the functions are not piecewise defined (as the more recent proposals discussed in the next section). Furthermore, the great symmetry of this model facilitates its phase plane analysis, which may be accomplished through lots of detailed phase diagrams from which we can infer KTz behavior (Tragtenberg and Yokoi, 1995; Kinouchi and Tragtenberg, 1996; Kuva et al., 2001; Copelli et al., 2004). 


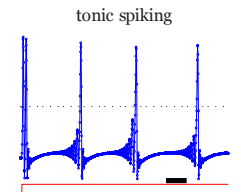

phasic bursting

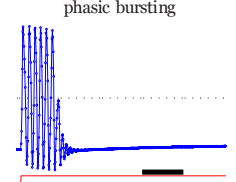

class 2 exc.

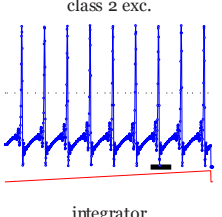

integrator

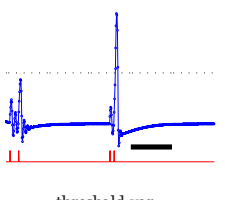

threshold var.

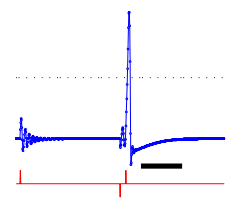

phasic spiking
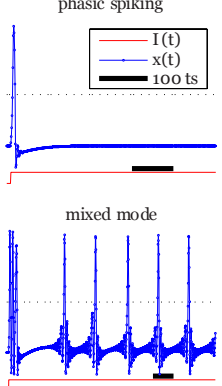

subthreshold osc.

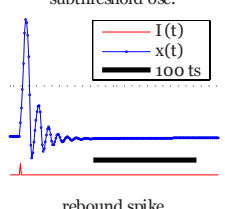

rebound spike

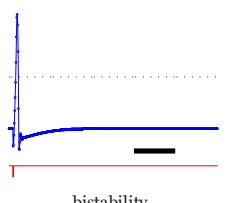

bistability

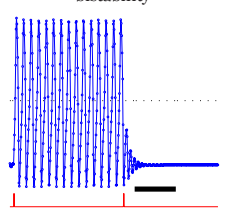

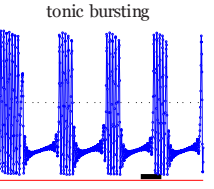

class 1 exc.

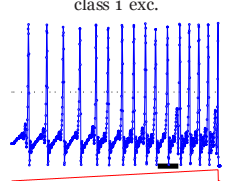

resonator

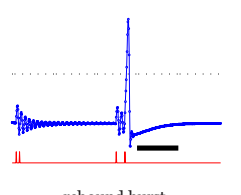

rebound burst

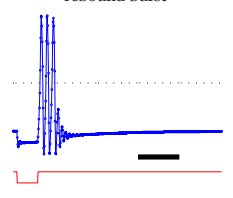

accomodation

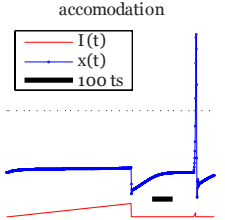

Figure 9: List of the KTz model excitable behaviors. The classification of these behaviors is based on Izhikevich and Hoppensteadt (2004).

\subsection{Recent proposals}

During the last decade, three other maps have been proposed: the Rulkov model (Rulkov, 2002), the Izhikevich model citepizhikevichMapas and the Courbage-Nekorkin-Vdovin model (Courbage et al., 2007). These and some other models have been reviewed elsewhere (Courbage and Nekorkin, 2009, Ibarz et al., 2011), so we are going to present only a brief description of the first two models because they are more easily found in literature. 


\subsubsection{The Rulkov Model}

The model proposed by Rulkov (2002) is, originally:

$$
\begin{aligned}
& x(t+1)=F(x(t), y(t)+\beta(t)) \\
& y(t+1)=y(t)-\mu(x(t)+1-\sigma(t))
\end{aligned},
$$

where $F(x, y)$ is a discontinuous function given by:

$$
F(x, y)= \begin{cases}\frac{\alpha}{1-x}+y & \text { if } x \leq 0 \\ \alpha+y & \text { if } 0<x<\alpha+y \\ -1 & \text { if } x \geq \alpha+y\end{cases}
$$

$\alpha$ and $\mu$ are adjustable parameters whilst $\beta(t)=\beta I(t)$ and $\sigma(t)=\sigma I(t)$ with $I(t)$ being the external input. When there is no external input, then $\beta(t)=\beta$ and $\sigma(t)=\sigma$ become adjustable parameters. The map consists of a fast dynamics in $x(t)$ and a slow dynamics in $y(t)$ when $\mu<<1$.

By the analysis of the nullclines of the fast and of the slow subsystems, one gets to the excitability threshold, $\sigma_{t h}$, in the $\alpha \times \sigma$ plane Rulkov (2002), which corresponds to a Subcritical Hopf Bifurcation:

$$
\sigma_{t h}=2-\sqrt{\alpha}
$$

Eq. 14 is plotted in Fig. 10 together with the limit between Bursting and Spiking phases (which was determined computationally by the author). There are also a Chaotic (Rulkov, 2001) and a Supercritical (Shilnikov and Rulkov, 2004) variant of the Rulkov model. The excitability of the latter is due to a Supercritical Hopf Bifurcation, hence its name.

\subsubsection{The Izhikevich Model}

Firstly proposed as an Ordinary Differential Equation (ODE) of continous time (Izhikevich, 2003), the map-based Izhikevich model is the following dis- 


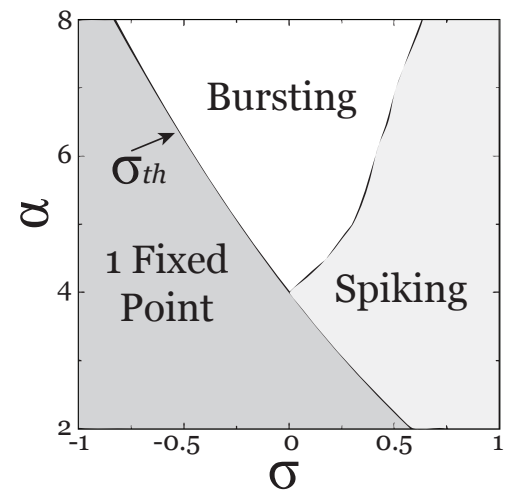

Figure 10: Bifurcation diagram of the Rulkov model (Eq. 12). The curve $\sigma_{t h}$ is given by Eq. 14. The stability limit between the phases of bursting and spiking is not well defined and has been determined computationally. Adapted from Rulkov (2002).

cretization (Izhikevich and Hoppensteadt, 2004):

$$
\begin{aligned}
& x(t+1)=0.04 x^{2}(t)+6 x(t)+140-y(t)+I(t) \\
& y(t+1)=a[b x(t)-y(t)]
\end{aligned}
$$

with the reset, if $x(t) \geq 30$ :

$$
\begin{aligned}
& x(t+1)=c \\
& y(t+1)=y(t)+d
\end{aligned},
$$

where $a, b, c$ and $d$ are the parameters, $I(t)$ is an input current (whether synaptic or external) and $t$ is the time in mili-seconds, assumed. The parameters $a$ and $b$ control balance of the slow dynamics of the in $y(t)$.

Fig. 11 shows the bifurcation diagram in the plane $c \times I$ for $a=0.02$, $b=0.25$ and $d=0$. The bifurcations undergone by the Izhikevich model depend strongly on parameters.

The author have proposed this model as a reduced Hodgkin-Huxley model by focusing on its bifurcations (Izhikevich, 2003), although the equations 


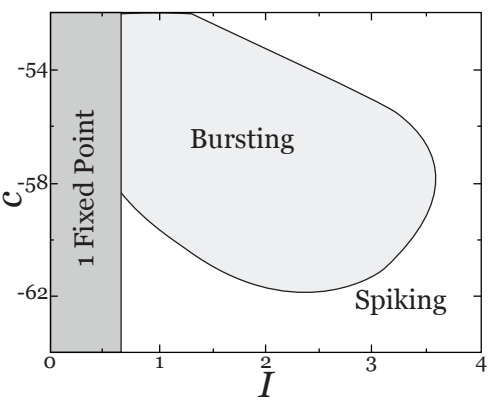

Figure 11: Bifurcation diagram of the Izhikevich model (Eq. 15) with $a=0.02, b=$ 0.25 and $d=0$. The separation between spiking and bursting have been determined computationally and is not well defined, thus we traced a representative line. Adapted from Ibarz et al. (2011).

resembles an Integrate-and-Fire (IF) model. Besides the behaviors presented in Fig. 9, this one also presents: spike frequency adaptation (similar to mixed mode panel), spike latency (a delayed response to a stimulus), depolarizing after-potential (the membrane potential is slightly depolarized right after the action potential), inhibitory induced spiking and inhibitory induced bursting (the onset of spiking or bursting during the injection of an inhibitory direct current) (Izhikevich and Hoppensteadt, 2004).

\section{Summary of the Addressed Models}

So far we have presented a description of the earliest maps proposed to describe neuronal activity together with some recent approaches. Izhikevich (2004) do an attempt to classify some of these models, comparing them with the biologically motivated models (HH-like neurons). To do so, he plots a graph with the amount of different biophysical behavior present in the 
model versus the amount of floating-point operations (FLOP - i.e. sums and multiplications) it would take for the computer to compute 1 ts of the model.

The Hodgkin-Huxley model may produce any biophysically plausible behavior, although it takes almost 100 times more FLOP. The Izhikevich model, with only 13 FLOP, may describe 20 different behaviors. Since the amount of FLOP is not a precise measure of the performance of any equation (as it discard anything else than sums and multiplications, like divisions, memory assignments, function calls, etc), we decided to do a benchmark test with the last three presented models by averaging the CPU time, $T$ in nanoseconds (ns), each model takes to perform a total of $T_{t s}=10000$ ts. We do that a number $S=10000 \times$ to reduce the variation of the measures. Thus, 1 ts takes, in average, $\bar{T}=T / T_{t s} / S$ real life nanoseconds to complete. The results are in Table 1 for codes running in 32 bits and 64 bits assemblies (both in a 64 bits CPU). In this table, the ellapsed time for each model, $T_{M}$, is calculated by $T_{M}=\tau T_{t s}$, where $\tau$ is the amount of miliseconds for each timestep of the considered model [ms/ts].

Changing from 32 bits to 64 bits makes the KTz neuron reduce by half its CPU time, reaching the other models' performance, whilst the others keep with their same CPU time. Nevertheless, one must remind that the choosing of the model depends strongly on the problem one is trying to study. For example, map-based neurons are more suitable for large-scale simulations or for generic studies (e.g. the cause of bifurcations, the rise of chaotic orbits, synchronization dynamics, avalanche dynamics, pattern recognition, memory 


\begin{tabular}{l|l|l|l|l} 
Model & $\bar{T}_{32 b i t}[\mathrm{~ns}]$ & $\bar{T}_{64 b i t}[\mathrm{~ns}]$ & $\tau[\mathrm{ms} / \mathrm{ts}]$ & $T_{M}[\mathrm{~ms}]$ \\
\hline \hline Izhikevich & $9.6 \pm 0.6$ & $10.7 \pm 0.7$ & $1^{*}$ & $10^{4}$ \\
Rulkov & $11.6 \pm 0.6$ & $11.5 \pm 0.5$ & 0.5 & $5 \times 10^{3}$ \\
KTz & $30.9 \pm 0.9$ & $18.9 \pm 0.7$ & 0.1 & $10^{3}$ \\
HH & $210 \pm 4$ & $255 \pm 5$ & 0.01 & $10^{2}$
\end{tabular}

Table 1: Comparison of CPU time, in nanoseconds [ns], of 1 ts for each map-based neuron model. We also present the CPU time of a HH-like neuron modeled by Cymbalyuk and Calabrese (2000) and integrated using a 4th order Runge-Kutta method. All these models are adjusted in bursting activity. Here, $\bar{T}$ is the CPU time (real life time), $\tau$ is the conversion factor between ms and ts and $T_{M}$ is the model ellapsed time. Conversion factors are from Izhikevich and Hoppensteadt (2004); Ibarz et al. (2011); Kuva et al. (2001). These factors generally take into consideration the empirical fact that 1 spike takes, approximately, $1 \mathrm{~ms} .{ }^{*}$ Sometimes, the Izhikevich conversion factor is $\tau=0.5$ $\mathrm{ms} / \mathrm{ts}$. 
modeling, etc); and biologically inspired models are more suitable for single cell studies (e.g. synaptic integration, dendritic cable filtering, effects of dendritic morphology, the dynamics of ionic currents, etc) or even smallscale network simulations - at least when there is worry with the simulation time.

Regardless of the chosen model and besides computational time and being a generic framework, maps bring many other advantages:

- There is no need for integration nor integration timestep adjustment; In fact, adjusting an integration timestep turns the ODE we are trying to solve into a discretized map. The bad thing in this approach is that the stability of the ODE may not correspond to the map stability.

- The solution is exact; When solving an ODE, the adjustment of an integration timestep turns the obtained solution in an approximation.

- They are more plausible than pure cellular automaton; The latter is also used to model neuronal activity (Copelli et al., 2002, 2005; Kinouchi and Copelli, 2006; Ribeiro et al., 2010), however it is a very abstract entity in which not only the time, but even the neuronal states are discretized.

- They keep the main biophysical properties of the Hodgkin-Huxley-like neurons, as we have seen in the previous section.

Thus, one is encouraged to choose the model which fits better to his purpose: choosing the best performance may make the phase plane analysis more difficult, as any of the Rulkov or Izhikevich model depends on a piecewise 
defined function. Or choosing simplicity over performance with the $\mathrm{KTz}$ model.

\section{Coupling Maps}

The modeling of neurons as maps is an important and active field of research in the past two decades, as we have seen in the previous sections of this paper. However, building up a network requires connecting the discrete time elements through synapses distributed along a particular network topology. This section is devoted to the synapse models. We summarize some types of connections, then we list some map models for them and eventually discuss the main behaviors of these models. We are aware of the risk of being repetitive, but we go through this for the sake of clarity and neatness.

In short, modeling synapses may or may not take into account the types of connections biologically observed. We can classify the simpler map-based synapse models in three cathegories: nonbiologically motivated, biologically motivated with diffusive (electrical) character and biologically motivated with impulsive (chemical) character.

The nonbiologically motivated models are usually rooted in the paradigm of coupled map lattices (Kaneko, 1993, Kaneko and Tsuda, 2001), which are not necessarily neuronal networks.

Diffusive (electrical) couplings are fast connections, also called gap junctions, that couple neighbouring neurons electrically in a direct form through channels. They are usually modelled by Ohm's law, where electric potential difference generates synaptic current through a time dependent (or not) conductance. 
Impulsive (chemical) synapses are connections with exchange of neurotransmitters and form the basis for neuronal communication (Keener and Sneyd, 1998). Phenomenological models or biological models describe the time dependence of the conductances, due to the release of the neurotransmitters.

In the next subsections, these three kind of models and their applications will be briefly reviewed. The neurons can also connect or disconnect as a function of time.This is called synapse plasticity and is an issue that will not be addressed in this paper.

\subsection{Types of Coupling}

\subsubsection{Nonbiologically Motivated Coupling}

A simplification of a neuronal network can be made if the neuron model is disconnected from biological background and the couplings between them are based in the paradigm of Coupled Map Lattices, i.e., the functions are not necessarily rooted on biological foundations.

The typical Coupled Map Lattices (CML) coupling of maps (given by Eq. 1) can be written in the following generalized mathematical form Ibarz et al., 2011):

$$
\vec{x}_{i}(t+1)=(1-\alpha) \vec{F}\left[\vec{x}_{i}(t)\right]+\frac{\alpha}{N_{i}} \sum_{j \neq i}^{N_{i}} \vec{F}\left[\vec{x}_{j}(t)\right],
$$

where $\vec{x}_{i}(t)$ is the vector state of the $i$ th neuron (node) at time $t$, with $1 \leq i \leq N, N$ being the total number of neurons, and $N_{i}$ is the total number of nodes connected to the $i$ th node. $\alpha \in[0 ; 1)$ is the coupling parameter: $\alpha=0$ means no coupling and $\alpha \rightarrow 1$ means that the node $i$ 's neighbors play a more important role to this element state than the node $i$ itself. 
Another kind of nonbiological coupling is to intertwin variables between elements of the network, like did Güémez and Matías (1996) with two chaotic Chialvo neurons - one of them drives the other - to study chaos supression:

$$
\begin{aligned}
& x_{1}(t+1)=\left[x_{1}(t)\right]^{2} \exp \left[y_{1}(t)-x_{1}(t)\right]+I \\
& y_{1}(t+1)=a y_{1}(t)-b x_{1}(t)+c \\
& x_{2}(t+1)=\left[x_{1}(t)\right]^{2} \exp \left[y_{2}(t)-x_{1}(t)\right]+I \\
& y_{2}(t+1)=a y_{2}(t)-b x_{2}(t)+c
\end{aligned}
$$

Notice that $x_{2}(t+1)$ is function of $x_{1}(t)$ and $y_{2}(t)$, and not of $x_{2}(t)$ as expected. Thus, it is said that the first neuron drives the second one. The synchronization may supress chaos in a couple of Chialvo neurons with intertwined variables.

It is important to notice that the framework of Eq. 17 does not hold if one chooses to work with more elaborate neuron models, like the KTz or the Rulkov models. The phase plane analysis and the bifurcation diagrams of such models provide a very specific way of inputing external currents into their equations, via the variable $I_{i}(t)$.

The so-called pulse-coupled neural network (PCNN) is an example of simple coupling through input currents. The input over neuron $i$ is assumed to be:

$$
I_{i}(t)=\sum_{j \neq i}^{N_{i}} J_{i j} x_{j}(t),
$$

where $J_{i j}$ is the coupling intensity (the conductance of the channel, in the case of a synapse). The sum runs over the $N_{i}$ neuron $i$ 's neighbors and the $x_{j}(t)$ 's are the presynaptic membrane potentials. It is called pulse coupling because, as soon as the neuron $j$ starts a pulse, it is readily transmitted to neuron $i$ scaled by the intensity $J_{i j}$ in the next time step. 
As examples of PCNN, take the Kinouchi and Tragtenberg (1996) approach to study emergence of collective oscillatory state: a fully connected network of KT neurons (Sec. 2.2) coupled via Eq. 19 homogeneously $\left(J_{i j}=\right.$ $\left.J_{j i}=J\right)$ and heterogeneously. The coupled model reads:

$$
\begin{aligned}
& x_{i}(t+1)=\tanh \left[\frac{x_{i}(t)-K y_{i}(t)+H+\sum_{j \neq i}^{N_{i}} J_{i j} x_{j}(t)}{T}\right] . \\
& y_{i}(t+1)=x_{i}(t)
\end{aligned}
$$

Another example is the chaotic Rulkov (2001) model in a mean field pulse-coupled network, implemented by the equation:

$$
\begin{aligned}
& x_{i}(t+1)=\frac{\alpha_{i}}{1+\left[x_{i}(t)\right]^{2}}+y_{i}(t)+\frac{\epsilon}{N} \sum_{j \neq i}^{N} x_{j}(t), \\
& y_{i}(t+1)=y_{i}(t)-\mu\left[x_{i}(t)-\sigma_{i}\right]
\end{aligned}
$$

used to study the synchronization of bursts when the network is not homogeneous (notice the indices in $\sigma_{i}$ and in $\alpha_{i}$ ). In this case, pulse coupling allows chaotic bursts to synchronize.

The next two kinds of coupling are biologically inspired and, hence, they are modeled by input currents just like the one used by PCNN.

\subsubsection{Diffusive Coupling}

Generally speaking, diffusive (electrical) couplings, also called gap junctions, are fast couplings, where channels between neighboring cells are formed and allow ions or small molecules to pass through them. Their exchange speed allow faster synchronization than chemical synapses. They are usual in the heart, other muscles and during development where synchronization play a major role (Keener and Sneyd, 1998), but in vertebrates they are a minority. 
Typically, they have the form (Connors and Long, 2004):

$$
I_{i}(t)=\sum_{j \neq i}^{N_{i}} J_{i j}\left[x_{j}(t)-x_{i}(t)\right],
$$

where $x_{i, j}$ are membrane potentials and $J_{i j}$ is the conductance of the gap junction channel.

\subsubsection{Impulsive Coupling}

The couplings via neurotransmitter exchanges are slower than the electrical ones, but more frequent in vertebrates. They are also called chemical of impulsive couplings and are responsible by the signal processing.

Generally, the synaptic current $I_{\text {syn }}(t)$ is given by

$$
I_{\text {syn }}(t)=g_{\text {syn }}(t)\left[x_{\text {post }}(t)-E_{\text {syn }}\right]
$$

where $g_{\text {syn }}(t)$ is the synaptic current, $x_{\text {post }}(t)$ the postsynaptic membrane potential, $E_{\text {syn }}$ is the reversal postsynaptic potential. The synaptic conductance may be modeled by a instantaneous rise with single exponential decay, an alpha function (with a continuous rise and fall) and a difference of exponentials (Roth and van Rossum, 2010).

Another punch line is the fast threshold modulation (FTM) approximation (Somers and Kopell, 1993), where there is a very sharp activation threshold and a constant conductance for each coupling such that

$$
I_{i}(t)=\sum_{k} I_{i, k}(t)
$$

where

$$
I_{i, k}(t)=g_{k}\left[x_{i}(t)-x_{r, k}\right] \sum_{k} H\left[x_{j}(t)-\theta_{k}\right]
$$


where $\mathrm{H}(\mathrm{x})$ is the step function, and the index $\mathrm{k}$ labels the different types of chemical synapses. The synaptic reversal potential is $x_{r, k}$ and $\theta_{k}$ are the presynaptic threshold of activation.

However, there is indeed a time scale (or more) for the synaptic coupling. Rulkov et al. (2004) and Bazhenov et al. (2005) propose an equation for the synaptic current:

$$
I_{\text {syn }}(t+1)=\gamma I_{\text {syn }}(t)-g\left[x_{\text {post }}-x_{r}\right] \delta\left(t-t_{\text {pre }, k}\right)
$$

where $\mathrm{g}$ is a conductance and $t_{p r e, k}$ is the time steps the presynaptic neuron has fired.

Kuva et al. (2001) proposed a map-based equation to model the chemical synaptic coupling between neurons. Their objective is to allow further research on Computational Neuroscience via biologically motivated Coupled Map Lattices. The input over neuron $i$ due to coupling current is the simple sum of the synaptic currents:

$$
I_{i}(t)=\sum_{j}^{N_{i}} Y_{i j}(t)
$$

where $N_{i}$ is the amount of neighbors of neuron $i$ and $Y_{i j}(t)$ is the synaptic current given by:

$$
\begin{aligned}
& Y_{i j}(t+1)=\left(1-\frac{1}{\tau_{1}}\right) Y_{i j}(t)+h_{i j}(t) \\
& h_{i j}(t+1)=\left(1-\frac{1}{\tau_{2}}\right) h_{i j}(t)+J_{i j} \Theta\left[x_{j}(t)\right]
\end{aligned},
$$

where $\tau_{1}$ and $\tau_{2}$ are exponential time constants, $J_{i j}$ is the coupling intensity (with dimension of conductance) and $\Theta(x)=1$ if $x>0$ and 0 otherwise is the Heaviside function. This synaptic current may be excitatory $(J>0)$ or inhibitory $(J<0)$. 
So far, notice that Eq. 28 is an approximation of a double exponential function, $f(t)=C\left[\exp \left(-t / \tau_{1}\right)-\exp \left(-t / \tau_{2}\right)\right]$, which reduces to an alpha function, $f(t)=C t \exp (-t / \tau)$, when $\tau_{1}=\tau_{2}=\tau ; C$ is a constant - see Fig. 12. Generally, this equation is used to model the conductance of synaptic currents. However, it is still a good approximation for modeling synaptic currents (de Schutter, 2010).

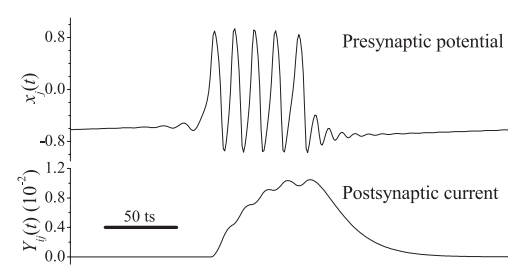

Figure 12: Time evolution of the postsynaptic current, Eq. 28 for $J=0.0001, \tau_{1}=\tau_{2}=15$ (bottom panel) due to a presynaptic KTz neuron in bursting regime, Eq. 11 with $K=0.6$, $T=0.35, \delta=\lambda=0.001$ and $x_{R}=-0.5$ (top panel).

Moreover one may question why there is no coupling parameter multiplying the synaptic current, $Y_{i j}(t)$, in Eq. 27. The coupling parameter is $J$. If one wish to multiply a new constant, say $C$, in that term: $C Y_{i j}(t)$, then a simple change of variables in Eq. 28 would vanish with $C$, keeping the same dynamical behavior: $Y_{i j}^{\prime}(t) \rightarrow C Y_{i j}(t), h_{i j}^{\prime}(t) \rightarrow C h_{i j}(t)$ and $J^{\prime} \rightarrow C J$.

Girardi-Schappo et al. (2012) studied a complete map-based neural network by using KTz maps coupled with Kuva synapses. The authors also adapted the Kuva synaptic current (Eq. 28), by adding a uniform random noise term in the coupling, in order to model synaptic noise observed experimentally (Peretto, 1994). Then, the Girardi-Schappo-Kinouchi-Tragtenberg 
(GKT) model is composed of Eq. 28 with $J_{i j} \equiv J_{i j}(t)$ given by:

$$
J_{i j}(t)=J+\epsilon_{i j}(t)
$$

where $J$ is the coupling parameter and $\epsilon_{i j}(t)$ is the uniform random time signal of amplitude $|R|$. The noise is different for every synapse $j \rightarrow i$. To keep the synaptic coherence (i.e. in order to the keep inhibitory synapses always inhibitting and excitatory synapses always exciting), the sign of $R$ is the same sign of $J$, then if $J>0$, we have $\epsilon_{i j}(t) \in[0 ; R]$, otherwise we have $\epsilon_{i j}(t) \in[-R ; 0]$. The noisy synaptic current is plotted in Fig. 13 .

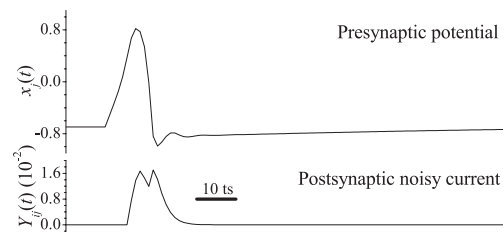

Figure 13: Time evolution of the postsynaptic noisy current, Eq. 28 with $J_{i j}(t)$ given by Eq. 29 with $J=0.001, R=0.01$ and $\tau_{1}=\tau_{2}=2$ (bottom panel) due to a presynaptic KTz neuron spike, Eq. 11 with $K=0.6, T=0.35, \delta=0.001, \lambda=0.008$ and $x_{R}=-0.7$ (top panel).

The important feature of this model is that the synapse is not always effective. It has a probability $q$ of exciting the postsynaptic neuron. Similar to the input current threshold, above which a neuron fires, there is a threshold in the coupling parameter, $J_{t h}$, above which the postsynaptic neuron will fire (the threshold $J_{t h}$ may also be negative when the postsynaptic neuron is excitable by rebound).

A first glance into Eq. 29 lead us to define the probability, $p$, of having 


$$
\left|J_{i j}(t)\right|>\left|J_{t h}\right|: \quad \quad p=\frac{J+R-J_{t h}}{R} .
$$

Note that $|J+R|>\left|J_{t h}\right|$ always, because $J, R$ and $J_{t h}$ have always the same sign. One could expect that $q=p$. That is not true because there is an internal dynamics in the synaptic current equations: as long as the spike takes place $\left(x_{j}(t)>0\right)$, the current keeps summing different quantities $J+\epsilon_{i j}(t)$. Thus, the same set $(J, R)$ may not always lead to a postsynaptic spike. Anyway, the quantity $p$ may be regarded as a first approximation to $q$.

\subsection{Applications of the synapse models}

A summary of the results obtained by each of the classes of synapse models (nonbiologically motivated, electric, chemical) in recognizing image patterns, modeling cat visual cortex, synchronization (connected with topology network, noise, spike-burst behavior, delay, rewiring, ...), bistable states of the brain, effects of external signals, information transmission and criticality in neuronal avalanches is given in this subsection.

\subsubsection{Nonbiologically motivated}

As one example of CML application is the study of synchonization made by Jampa et al. (2007). They connected Chialvo neurons through a short version of Eq. 17 , in which the coupling term is written only as $(\epsilon / 2)\left[x_{j(t)}(t)+\right.$ $\left.x_{k(t)}(t)\right]$, where the indices $j(t)$ and $k(t)$ are picked at each timestep, with probability $p$, by randomly assigning to each node an index, $l \in[1 ; N]$, and then calculating $j(t)=l-1$ and $k(t)=l+1$ (modulo $N)$. The rewiring probability $p$ and the coupling strength $\epsilon$ determine the kind of synchronization: 
chaotic, spatio-temporal chaos or fixed point.

On the other hand, the PCNN approach is used for many purposes: modeling cat visual cortex(Eckhorn et al., 1990), recognizing image patterns (Wang et al. 2010), synchronization and related problems (Kinouchi and Tragtenberg, 1996; Goel and Ermentrout, 2002; Zou et al., 2009).

Eckhorn et al. (1990) modeled the cat visual cortex via PCNN, particularly the stimulus-induced oscillations, used for pattern recognition and feature associations. Wang et al. (2010) reviewed the successful use of PCNN in many dimensions of image processing: segmentation, denoising, object and edge detection, feature extraction and pattern recognition (enhancement, fusion, etc). Goel and Ermentrout (2002) discretized an ODE, and found waves in a ring and synchonization stability dependent of the number of neurons. Stable chaos was found in a PCNN of discontinuous maps by Zou et al. (2009).

Pontes et al. (2008) found that the coupling strength needed to synchronized Rulkov bursters is smaller when the range of couplings is bigger.

de Vries (2001a b) showed that mean field-coupling of the same system can turn isolated spiking into coupled bursting neurons. Then, in this case bursting can be seen as an emergent behavior.

de Vries (2012a) used coupled logistic maps, as well as Stuart-Landau oscillators and leaky IF neurons, to study dynamical properties of coupled oscillator sparse lattices in many scales of length. They show a transition from micro to macroscopic scale in network syncronization driven by a critical conectivity.

López-Ruiz and Fournier-Prunaret (2012) mimicked a bistable (sleep- 
awaken) brain by a logistic map lattice, with usual CML interactions.

The pulse-coupled KT model with homogeneous couplings, $J_{i j}=J$, may lead a network of silent neurons (adjusted with $\left(K, T, H<H_{s}\right)-$ Eq. 8 , outside of the bubble in Fig. 2 into a collective oscillating phase if the sum $I_{i}(t)=J \sum_{\langle j\rangle} x_{j}(t)$ is sufficiently big - such that $\tilde{H}=H+I_{i}(t)>H_{s}(K, T)$ (for neurons with $H<0$ ).

More clearly, if $H<0$, the fixed point is also $x^{*}<0$. Thus, if $J<0$ (inhibitory coupling), the sum $I_{i}(t)=J \sum_{\langle j\rangle} x_{j}(t)$ will be a positive number and the achieved state will have a new $\tilde{H}$ given by $\tilde{H}=H+I_{i}(t)$. Then, there is a threshold - exactly equal to the excitability threshold discussed in Sec. 2.2 - above which the network will spontaneously oscillate, even though any uncoupled neuron is silent (in the fixed point). This is a counter-intuitive result, although it also happens with realistic neurons Golomb and Rinzel, 1993; Ernst et al., 1995).

If the term $|J|$ is big enough, the network will cross through the bubble in Fig. 2 and will reach the upper fixed point, $x^{*}>0$. This picture may be generalized to the heterogeneous case, however the value of $I_{i}(t)$, in such case, is not guaranteed to be positive.

The heterogeneous case may be used both for modeling practical problems and for investigation purposes. As examples, one could assemble a neural network for pattern recognition by mixing linear neurons (in the output) and KT units and adjusting weights according to a training algorithm Kinouchi and Tragtenberg, 1996) (see Albano et al. (1992) for an application with neurons given by Eq. 2, but with a nonlinear activation function). On the other hand, Izhikevich (2003) studies brain rythms via a PCNN made of his 
neuron model (discretized with a $0.1 \mathrm{~ms}$ timestep).

The Izhikevich heterogeneous network consists in mixed excitatory $\left(J_{i j}>\right.$ $0)$ and inhibitory $\left(J_{i j}<0\right)$ coupling. From the network's time evolution, emerge groups of synchronized neurons in different phases - the so called polychronous state. Izhikevich (2006) discuss that these polychronous groups represent the memory of the network, as different external stimuli produce different polychronization patterns. Yet, the number of produced patterns is far greater than the number of neurons in the network, yielding a very big memory capacity.

\subsubsection{Diffusive coupling}

Diffusive coupling has been extensively used in the study of synchronization, spatio-temporal chaos, effects of external inputs on a neuronal network, spiking-bursting transition, among other subjects.

Rulkov (2002) studied the existence of synchronization regimes for spiking and bursting activities of rulkov-map a function of neuron coupling strength.

de Vries (2012b) connected Rulkov neurons in a small-world network through typical electrical connections to study synchronization and spatiotemporal chaos.

Subthreshold stimulus were found to induce synchronization in a noisy square lattice Rulkov network by Wang et al. (2007). The study of Rulkov neurons with information transmission delay in a small-world geometry with additive noise and delay, as done by Wang et al. (2008), show the existence of transitions in synchronization as a function of the delay. The delay may also induce stochastic resonance in a scale-free network of Rulkov neurons, have shown Wang et al. (2009). 
The increasing of diversity of Rulkov neuron connections may induce synchronization in a Rulkov map neural network, as analyzed by Chen and Liu (2009). The influence of a mix of electrical and chemical synapses connecting Rulkov neurons on burst synchonization and transmission (seen as chaotic itinerancy Kaneko and Tsuda (2003)) were studied by Tanaka et al. (2006) . Ivanchenko et al. (2007) and Ivanchenko et al. (2004) studied transitions between bursting and spiking Rulkov neurons connected by electrical synapses in many time scales and the chaotic phase synchronization by an external periodic input to a single neuron. Batista et al. (2007) and Batista et al. (2009) also showed that external periodic signals can generate burst synchronization in networks of Rulkov neurons electrically coupled.

\subsubsection{Impulsive coupling}

Connecting map neurons by chemical synapses is not as popular as using electrical synapses, despite the chemical are more widespread than the electrical. One of the most popular approaches is the FTM, which shows no synaptic dynamics. However, there are some proposals of describing the chemical synapse dynamics applied to network behavior.

In the FTM front, Ivanchenko et al. (2007) showed that electrical and impulsive (chemical) synapses behave like electrical, since networks of Rulkov maps exhibit transition syncronized-desynchronized transition as a function of the coupling strength.

Ibarz et al. (2008) used FTM to show a bunch of effects of impulsive synapses: excitatory synapses may generate antiphase synchronization, synapse may change from excitatory to inhibitory as a change in conductance and with the same reversal potential, small variations in the synaptic threshold 
may cause big changes in synchronization of spikes within bursts.

Shi and Lu (2009) also applied FTM coupling in the studiyg of in-phase burst synchronization of Rulkov neurons.

Inhibitory bursting Rulkov maps chemically coupled may exhibit powerlaw behavior at the onset of a synchronization transition, driven by the coupling strength or stimulation current( Franovic and Miljkovic, 2010).

The effect of delay in in-phase and anti-phase synchronization was the object of Franovic and Miljkovic (2011) in Rulkov map neural network connected by reciprocal sigmoid chemical synapses.

Another different approach is to consider connections with their own dynamics. Rulkov et al. (2004) proposed a chemical synapse model, given by

$$
I_{\text {syn }}(t+1)=\gamma I_{\text {syn }}(t)-g_{\text {syn }}\left[x_{\text {post }}(t)-x_{r}\right] \delta\left(t-t_{\text {pre }, k}\right)
$$

where the delta function is 1 for a presynaptic spike and 0 otherwise. $\gamma$ is a decay constant, for an single exponential dynamics in synapse. The model exhibit many dynamic behaviors and is qualitatively comparable to Hodgkin-Huxley model. Using basically the same model, Bazhenov et al. 2005) determined resonance properties on collective behavior in a cortical network model containing excitatory and inhibitory cells and showed that network interactions can enhance the frequency range of reliable responses, such that the latter can be controlled by the strength of synaptic connections.

Dynamical map models for impulsive synapses were also proposed by Kuva et al. (2001) and Girardi-Schappo et al. (2012). The first one uses uniform synapses and the second introduces synaptic noise, believed to be crucial to information processing. With their model, Girardi-Schappo et al. (2012) could study the effects of the synaptic inefficacy makes a network of 
neurons coupled with GKT synapses a matter of stochastic dynamics, since the amount of neurons, $s$, that will fire in the network due to an initial stimulation is not obvious. The network activity due to one stimulus is called an avalanche. The authors investigated regular square lattices with free boundary conditions of KTz neurons coupled with GKT synapses and found that $s$ follows a powerlaw $P(s) \sim s^{-\mu}$ with $\mu=1.35$. Besides, the duration of avalanches, $w$, also follows a powerlaw $P(w) \sim w^{-\nu}$, with $\nu=1.50$.

Moreover, the authors measured the activity of a subsampled network - a network in which the measurement can be made in only a very small fraction of the elements. Generally, experimental setups are subsampled, since the number of neurons which are recorded is far less than the number of neurons in the sample (Priesemann et al., 2009, Ribeiro et al., 2010). The subsampling results matched the experiments of Ribeiro et al. (2010) and, together with the powerlaw behavior in the network activity, both in temporal and spatial dimensions, show that the system is critical and suggests that there may be a Self-Organized Critical (SOC) state in brain activity and neural network models (Girardi-Schappo et al. 2012).

The SOC state is, generally, present when there is a balance between tensioning and relaxing the system (Bak et al., 1987, 1988, Christensen et al., 1993; Jensen, 1998; Dhar, 2006; Bonachela and Muñoz, 2009, and many others). Due to theoretical considerations about criticality in neural networks (Usher et al., 1995; Stassinopoulos and Bak, 1995; Herz and Hopfield, 1995), Beggs and Plenz (2003) proposed SOC to explain the brain activity. Avalanches are experimentally observed (Beggs and Plenz, 2003, 2004, Priesemann et al., 2009; Ribeiro et al., 2010, Chialvo, 2010, Werner, 2010, 
Beggs and Timme, 2012; Shew and Plenz, 2013, and references there in), although there is a great debate on the criticality of the brain.

The basic idea is that the brain works with the precisely needed amount of activity and that there may be a homeostatic mechanism which led the brain to such a state. Within this framework, mental diseases and disorders would be associated with deviations from the critical amount of activity (Chialvo, 2004, 2010; Vertes et al., 2011). The critical state has been shown to enhance the dynamical range of sensitivity to external stimuly Kinouchi and Copelli, 2006, Shew et al., 2009), to optimize the memory and learning processes (de Arcangelis et al. 2006) and the computational power of the brain (Werner, 2010; Shew and Plenz, 2013; Beggs and Timme, 2012).

A necessary condition for SOC is that the system may not be imposed into the critical state, rather it should find its own path towards criticality with no fine tuning. As no computer model could work on its own, without external tuning of parameters, Kinouchi (1998) proposed to call these systems SelfOrganized quasi-Critical ( $\mathrm{SOqC})$. $\mathrm{SOqC}$ systems have a balance dynamical equation that makes the system oscillate in the neighborhood of the critical point. In this sense, the GKT model is not SOC, nor $\mathrm{SOqC}$, because the authors had to adjust the parameters $J$ and $R$. However, a variation of the GKT model could contain a homeostatic mechanism over the parameter $R$ or $J$, for instance, that would take the system towards the critical point, turning the system into a $\mathrm{SOqC}$ system. 


\section{Concluding Remarks}

Although generally studied in the context of dynamical systems, some of the models outlined in this work may be used in computational applications, such as pattern recognition, data analysis, data classification, data association and so on, like the McCulloch-Pitts model utilized in the perceptron. We directed our studies mainly through the historical development of the map-based models, by theoretically constructing over the McCulloch-Pitts model, adding delayed self-couplings (which creates dynamical behaviors), changing to a continuous activation function and adding coupling currents to the model.

This approach allowed us to close the gap between the first discrete time models and the most recent maps. In general, we tried to keep the original design concepts of the models, except for the Izhikevich's, which was originally designed as an ODE. Nevertheless we assume that the discretization of an ODE results in a map, with behavior of its own. Thus, we linked two entire family of models with very rich excitable and dynamical behaviors, namely the perceptron family (from McCulloch-Pitts until KTz) and the HH family (from Chialvo to Izhikevich). We also showed that there is a close dynamical correspondence between $\mathrm{KTz}$ and the behaviors of $\mathrm{HH}$.

The models of the HH family are, generally, piecewise defined functions. However, all of these models present bursting activity with only two dynamical variables, differently from the $\mathrm{KTz}$ map (whose bursting behavior was based in Hindmarsh-Rose model). On the other hand, the symmetry and

simplicity of the KTz map may be of great help during the phase plane and bifurcation analysis. KT and KTz also may be suitable when there is a need 
to explore the effects of the whole action potential, as all the other models assume that the spike occurs instantly. Therefore, if one wants to model a more rigorous synaptic transmission with the map framework, $\mathrm{KT}$ or $\mathrm{KTz}$ are should be chosen.

Every map model presents a similar computational performance, as discussed in Sec. 3, which is approximately $20 \times$ faster than the HH performance. Thus, the most prominent features of all the studied models are their simiplicity, reliability, numerical stability and computational performance.

Moreover, we categorized the many types of coupling used to connect the covered models. Many times, the coupling and the network topology are indissociable, forcing one to study the coupling of neurons under a given topology. From pulse-coupled networks to map-based modeling of chemical synapses, we presented their most common usability, relating with the results achieved in each case. Since synapses is, alone, a broad research area, we highlight the importance of modeling synapses specifically for working with map-based neural networks, as did Kuva et al. (2001), otherwise the differential equations would have to be discretized for each case, bringing additional modeling problems, namely how to synchronize the time evolution of the map-based neuron with the time evolution of the synapses, as the latter should be precisely solved to avoid numerical inaccuracy?

Finally, recent results have shown that map-based neural networks is a promising developing field, for example, Girardi-Schappo et al. (2012) modeled both synapses and neurons with maps and showed that a critical state may develop in regular lattices when the coupling is subjected to uniform noise. Just as examples, on the list of some still unexplored paths in map- 
based neuronal modeling is the compartimental modeling and the seek for a canonical purely map-based model (in the ODE framework, the neuron model differs from the whole class of HH-type neurons by just a change o variables (Hoppensteadt and Izhikevich, 2002); on the other hand, a canonical map-based model would be the most simple model capable of describing all the HH-type neurons behaviors).

With this work, we hope we have brought more attention to this kind of modeling, which may play an important role in the forthcoming years, both in technologic applications and in neuroscientific research.

\section{Acknowledgement}

We would like to thank the invitation to make this neuron map modeling overview from Antonio Carlos Roque da Silva.

\section{References}

Aihara, K., Suzuki, H., 2010. Theory of hybrid dynamical systems and its applications to biological and medical systems. Phil. Trans. R. Soc. A 368, 4893-4914.

Aihara, K., Takabe, T., Toyoda, M., 1990. Chaotic neural networks. Phys. Lett. A 144(6,7), 333-340.

Albano, A. M., Passamante, A., Hediger, T., Farrell, M. E., 1992. Using neural nets to look for chaos. Physica D 58, 1-9.

Azevedo, F. A. C., Carvalho, L. R. B., Grinberg, L. T., Farfel, J. M., Ferretti, R. E. L., Leite, R. E. P., Filho, W. J., Lent, R., Herculano-Houzel, S., 2009. 
Equal numbers of neuronal and nonneuronal cells make the human brain an isometrically scaled-up primate brain. J. Comp. Neurol. 513, 532-541.

Bak, P., Tang, C., Wiesenfeld, K., 1987. Self-organized criticality: An explanation of 1/f noise. Phys. Rev. Lett. 59(4), 381-384.

Bak, P., Tang, C., Wiesenfeld, K., 1988. Self-organized criticality. Phys. Rev. A $38(1), 364-374$.

Batista, C. A. S., Batista, A. M., de Pontes, J. A. C., Lopes, S. R., Viana, R. L., 2009. Bursting synchronization in scale-free networks. Chaos, Solitons and Fractals 41, 2220-2225.

Batista, C. A. S., Batista, A. M., de Pontes, J. A. C., Viana, R. L., Lopes, S. R., 2007. Bursting synchronization in scale-free networks. Phys. Rev. E $76,016218$.

Bazhenov, M., Rulkov, N. F., Fellous, J., Timofeev, I., 2005. Role of network dynamics in shaping spike timing reliability. Phys. Rev. E 72, 041903.

Beggs, J. M., Plenz, D., 2003. Neuronal avalanches in neocortical circuits. J. Neurosci. 23(35), 11167-11177.

Beggs, J. M., Plenz, D., 2004. Neuronal avalanches are diverse and precise activity patterns that are stable for many hours in cortical slice cultures. J. Neurosci. 24(22), 5216-5229.

Beggs, J. M., Timme, N., 2012. Being critical of criticality in the brain. Front. Physiol. 3, 163. 
Bellman, R. E., 2003. Dynamic Programming. Dover.

Bonachela, J. A., Muñoz, M. A., 2009. Self-organization without conservation: true or just apparent scale-invariance? J. Stat. Mech., P09009.

Bower, J. M., Beeman, D., 2003. The Book of GENESIS: Exploring Realistic Neural Models with the GEneral NEural SImulation System. Internet Edition.

Caianello, E. R., 1961. Outline of a theory of thought process and thinking machines. J. Theor. Biol. 1, 204-235.

Carnevale, N. T., Hines, M. L., 2006. The NEURON Book. Cambridge University Press.

Chen, H., Liu, J. Z. J., 2009. Enhancement of neuronal coherence by diversity in couple rulkov-map models. Physica A 19, 023112.

Chialvo, D. R., 1995. Generic excitable dynamics on a two-dimensional map. Chaos Solitons Fractals 5, 461-479.

Chialvo, D. R., 2004. Critical brain networks. Physica A 340, 756-765.

Chialvo, D. R., 2010. Emergent complex neural dynamics. Nat. Phys. 6, 744750 .

Christensen, K., Flyvbjerg, H., Olami, Z., 1993. Self-organized critical forestfire model: Mean-field theory and simulation results in 1 to 6 dimensions. Phys. Rev. Lett. 71(17), 2737-2740. 
Connors, B. W., Long, M. A., 2004. Electrical synapses in the mammalian brain. Annual Review of Neuroscience 27, 393-418.

Copelli, M., Oliveira, R. F., Roque, A. C., Kinouchi, O., 2005. Signal compression in the sensory periphery. Neurocomputing 65-66, 691-696.

Copelli, M., Roque, A. C., Oliveira, R. F., Kinouchi, O., 2002. Physics of psychophysics: Stevens and weber-fechner laws are transfer functions of excitable media. Phys. Rev. E 65, 060901.

Copelli, M., Tragtenberg, M. H. R., Kinouchi, O., 2004. Stability diagrams for bursting neurons modeled by three-variable maps. Physica A 342, 263269 .

Courbage, M., Nekorkin, V. I., 2009. Map based models in neurodynamics. Int. J. Bifurcat. Chaos 20(6), 1631-1651.

Courbage, M., Nekorkin, V. I., Vdovin, L. V., 2007. Chaotic oscillations in a map-based model of neural activity. Chaos 17(4), 043109.

Cymbalyuk, G., Calabrese, R. L., 2000. Oscillatory behaviors in pharmacologically isolated heart interneurons from the medicinal leech. Neurocomputing 32-33, 97-104.

Dayan, P., Abbott, L. F., 2001. Theoretical Neuroscience: Computational and Mathematical Modeling of Neural Systems. The MIT Press.

de Arcangelis, L., Perrone-Capano, C., Herrmann, H. J., 2006. Self-organized criticality model for brain plasticity. Phys. Rev. Lett. 96, 028107. 
de Schutter, E. (Ed.), 2010. Computational Modeling Methods for Neurocientists. The MIT Press.

de Vries, G., 2001a. Bursting as an emergent phenomenon in coupled chaotic maps. Phys. Rev. E 64, 051914.

de Vries, G., 2001b. From spikers to bursters via coupling: help from heterogeneity. Bull. Math. Biol 63, 371-391.

de Vries, G., 2012a. Collective dynamics in sparse networks. Phys. Rev. Lett. 109, 138103.

de Vries, G., 2012b. Collective dynamics in sparse networks. Phys. Rev. Lett. 109, 138103.

Dhar, D., 2006. Theoretical studies of self-organized criticality. Physica A $369,29-70$.

Eckhorn, R., Reitboeck, H. J., Arndt, M., Dicke, P. W., 1990. Feature linking via synchronization among distributed assemblies: simulations of results on cat visual cortex. Neural Computation 2, 293-307.

Ernst, U., Pawelzik, K., Geisel, T., 1995. Synchronization induced by temporal delays in pulse-coupled oscillators. Phys. Rev. Lett. 74, 1570-1573.

FitzHugh, R., 1955. Mathematical models of threshold phenomena in the nerve membrane. Bulletin of Mathematical Biophysics 17, 257-278.

Franovic, I., Miljkovic, V., 2010. Power law behavior related to mutual synchronization of chemically coupled map neurons. Euro. Phys. J. B 76, 613-624. 
Franovic, I., Miljkovic, V., 2011. The effects of synaptic time delay on motifs of chemically coupled rulkov model neurons. Commun. Nonlinear Sci. Numer. Simul. 16, 623-633.

Girardi-Schappo, M., Kinouchi, O., Tragtenberg, M. H. R., 2012. Critical avalanches and subsampling in map-based neural networks. arXiv:1209.3271 [cond-mat.dis-nn].

Goel, P., Ermentrout, B., 2002. Synchrony, stability, and firing patterns in pulse-coupled oscillators. Physica D 163, 191-216.

Golomb, D., Rinzel, J., 1993. Dynamics of globally coupled inhibitory neurons with heterogeneity. Phys. Rev. E 48, 4810-4814.

Güémez, J., Matías, M. A., 1996. Synchronous oscillatory activity in assemblies of chaotic model neuron. Physica D 96, 334-343.

Herz, A. V. M., Hopfield, J. J., 1995. Earthquake cycles and neural reverberations: Collective oscillations in systems with pulse-coupled threshold elements. Phys. Rev. Lett. 75, 1222-1225.

Hindmarsh, J. L., Rose, R. M., 1984. A model of neuronal bursting using three coupled first order differential equations. Proc. R. Soc. Lond., B, Biol. Sci. 221, 87-102.

Hopfield, J. J., 1984. Neurons with graded response have collective computational properties like those of two-sate neurons. Proc. Nat. Acad. Sci. (USA) 81, 3088-3092. 
Hoppensteadt, F. C., Izhikevich, E. M., 2002. Canonical Neural Models. The MIT Press.

Ibarz, B., Cao, H., Sanjuan, M. A. F., 2008. Bursting regimes in map-based neuron models coupled through fast threshold modulation. Phys. Rev. E 77, 051918.

Ibarz, B., Casado, J. M., Sanjuán, M. A. F., 2011. Map-based models in neuronal dynamics. Phys. Rep. 501, 1-74.

Ivanchenko, M. V., Osipov, G. V., Shalfeev, V. D., Kurths, J., 2004. Phase synchronization in ensembles of bursting oscillators. Phys. Rev. Lett. 93, 134101.

Ivanchenko, M. V., Osipov, G. V., Shalfeev, V. D., Kurths, J., 2007. Network mechanism for burst generation. Phys. Rev. Lett. 98, 108101.

Izhikevich, E. M., 2003. Simple model of spiking neurons. IEEE Trans. Neural Netw. 14(6), 1569-1572.

Izhikevich, E. M., 2004. Which model to use for cortical spiking neurons? IEEE Trans. Neural Netw. 15, 1063-1070.

Izhikevich, E. M., 2006. Polychronization: Computation with spikes. Neural Comput. 18(2), 245-282.

Izhikevich, E. M., Hoppensteadt, F., 2004. Classification of bursting mappings. Int. J. Bifurcat. Chaos 14(11), 3847-3854.

Jampa, M. P. K., Sonawane, A. R., Gade, P. M., Sinha, S., 2007. Sincronization in a network of model neurons. Physical Review E 75, 026215. 
Jensen, H. J., 1998. Self-Organized Criticality: Emergent Complex Behavior in Phyical and Biological Systems. Cambridge University Press.

Kaneko, K., 1993. Theory and Applications of Coupled Map Lattices. Wiley.

Kaneko, K., 1994. Relevance of dynamic clustering to biological networks. Physica D 75, 55-73.

Kaneko, K., Tsuda, I., 2001. Complex Systems: Chaos and Beyond. A Constructive Approach with Applications in Life Sciences. Springer.

Kaneko, K., Tsuda, I., 2003. Chaotic itinerancy. Chaos 13, 926-936.

Keener, J., Sneyd, J., 1998. Mathematical Physiology. Springer.

Kinouchi, O., Fevereiro 1998. Self-organized (quasi-)criticality: the extremal feder and feder model. arXiv:cond-mat/9802311v1.

Kinouchi, O., Copelli, M., 2006. Optimal dynamical range of excitable networks at criticality. Nat. Phys. 2, 348-351.

Kinouchi, O., Tragtenberg, M. H. R., 1996. Modeling neurons by simple maps. Int. J. Bifurcat. Chaos 6, 2343-2360.

Kuva, S. M., Lima, G. F., Kinouchi, O., Tragtenberg, M. H. R., Roque, A. C., 2001. A minimal model for excitable and bursting elements. Neurocomputing 38-40, 255-261.

Little, W. A., 1974. The existence of persistent states in the brain. Math. Biosci. 19, 101-120. 
López-Ruiz, R., Fournier-Prunaret, D., August 2012. The bistable brain: a neuronal model with symbiotic interactions. arXiv:nlin.CD/1208.0223v1.

McCulloch, W. S., Pitts, W. H., 1943. A logical calculus of the ideas immanent in nervous activity. Bull. Math. Biophys. 5, 115-133.

Morris, C., Lecar, H., 1981. Voltage oscillations in the barnacle giant muscle fiber. Biophysics Journal 35, 193-213.

Nagumo, J., Arimoto, S., Yoshizawa, S., 1962. An active pulse transmission line simulating nerve axon. Proceedings of the IRE 50, 2061-2070.

Nagumo, J., Sato, S., 1972. On a response characteristic of a mathematical neuron model. Kybernetik 10, 155-164.

Pasemann, F., 1993. Dynamics of a single model neuron. Int. J. Bifurcat. Chaos 2, 271-278.

Pasemann, F., 1997. A simple chaotic neuron. Physica D 104, 205-2011.

Peretto, P., 1994. An Introduction to the Modeling of Neural Networks. Cambridge University Press.

Pontes, J. C. A., Viana, R. L., Lopes, S. R., Batista, C. A. S., Batista, A. M., 2008. Bursting synchronization in non-locally coupled maps. Physica A $387,4417-4428$.

Priesemann, V., Munk, M. H. J., Wibral, M., 2009. Subsampling effects in neuronal avalanche distributions recorded in vivo. BMC Neurosci. 10, 40. 
Ribeiro, T. L., Copelli, M., Caixeta, F., Belchior, H., Chialvo, D. R., Nicolelis, M. A. L., Ribeiro, S., 2010. Spike avalanches exhibit universal dynamics across the sleep-wake cycle. PLoS ONE 5(11), e14129.

Roth, A., van Rossum, M. C. W., 2010. Modeling Synapses. The MIT Press.

Rulkov, N. F., 2001. Regularization of synchronized chaotic bursts. Phys. Rev. Lett. 86, 183-186.

Rulkov, N. F., 2002. Modeling of spiking-bursting neural behavior using twodimensional map. Phys. Rev. E 65, 041922.

Rulkov, N. F., Timofeev, I., Bazhenov, M., 2004. Oscillations in large-scale cortical networks: map-based model. J. Comput. Neurosci. 17, 203-223.

Shew, W. L., Plenz, D., 2013. The functional benefits of criticality in the cortex. Neuroscientist 19(1), 88-100.

Shew, W. L., Yang, H., Petermann, T., Roy, R., Plenz, D., 2009. Neuronal avalanches imply maximum dynamic range in cortical networks at criticality. J. Neurosci. 29(49), 15595-15600.

Shi, X., Lu, Q., 2009. Burst synchronization of electrically and chemically coupled map neurons. Physica A 388, 2410-2419.

Shilnikov, A. L., Rulkov, N. F., 2004. Subthreshold oscillations in a mapbased neuron model. Phys. Lett. A 328, 177-184.

Somers, D., Kopell, N., 1993. Rapid synchronization through fast threshold modulation. Biol. Cybern. 68, 393-407. 
Stassinopoulos, D., Bak, P., 1995. Democratic reinforcement: A principle for brain function. Phys. Rev. E 51(5), 5033-5039.

Tanaka, G., Ibarz, B., Sanjuán, M. A. F., Aihara, K., 2006. Synchronization and propagation of bursts in networks of coupled map neurons. Chaos 16, 013113.

Tragtenberg, M. H. R., Yokoi, C. S. O., 1995. Field behavior of an ising model with competing interactions on the bethe lattice. Phys. Rev. E 52(3), 21872197.

Usher, M., Stemmler, M., Olami, Z., 1995. Dynamic pattern formation leads to $1 / \mathrm{f}$ noise in neural populations. Phys. Rev. Lett. 74, 326-329.

Veiga, F. L. S., Tragtenberg, M. H. R., 2001. A very stochastic resonant neuron model. Neurocomputing 38-40, 423-431.

Vertes, P. E., Bassett, D. S., Duke, T., 2011. Scale-free statistics of neuronal assemblies predict learning performance. BMC Neurosci. 12(Suppl 1), O4.

Wang, Q. Y., Duan, Z., Perc, M., Chen, G. R., 2008. Synchonization transitions on small-world neuronal networks: effects of information transmission delay and rewiring probability. Europhys. Lett. 83, 50008.

Wang, Q. Y., Lu, Q. S., Chen, G. R., 2007. Subthreshold stimulus-aided temporal order and synchronization in a square lattice noisy neuronal network. Europhys. Lett. 77, 10004.

Wang, Q. Y., Perc, M., Duan, Z., Chen, G. R., 2009. Delay-induced multiple stochastic resonances on scale-free neuronal networks. Chaos 19, 023112. 
Wang, Z., Ma, Y., Cheng, F., Yang, L., 2010. Review of pulse-coupled neural networks. Image Vis. Comput. 28, 5-13.

Werner, G., 2010. Fractals in the nervous system: conceptual implications for theoretical neuroscience. Front. Physiol. 1, 15.

Yokoi, C. S. O., de Oliveira, M. J., Salinas, S. R., 1985. Strange attractor in the ising model with competing interactions on the cayley tree. Phys. Rev. Lett. 54(3), 163-166.

Zou, H., Guan, S., Lai, C.-H., 2009. Dynamical formation of stable irregular transients in discontinuous map systems. Phys. Rev. E 80, 046214. 